\title{
CYP450 phenotyping and metabolite identification of quinine by accurate mass UPLC-MS analysis: a possible metabolic link to blackwater fever
}

\author{
Sean R Marcsisin ${ }^{1}$, Xiannu Jin ${ }^{1}$, Theresa Bettger ${ }^{1}$, Nicholas McCulley ${ }^{1}$, Jason C Sousa', G Dennis Shanks², \\ Babu L Tekwani ${ }^{3}$, Rajnish Sahu ${ }^{3}$, Gregory A Reichard ${ }^{1}$, Richard J Sciotti ${ }^{1}$, Victor Melendez ${ }^{1}$ and Brandon S Pybus ${ }^{1 *}$
}

\begin{abstract}
Background: The naturally occurring alkaloid drug, quinine is commonly used for the treatment of severe malaria. Despite centuries of use, its metabolism is still not fully understood, and may play a role in the haemolytic disorders associated with the drug.

Methods: Incubations of quinine with CYPs 1A2, 2C9, 2C19, 2D6, and 3A4 were conducted, and the metabolites were characterized by accurate mass UPLC-MS ${ }^{\mathrm{E}}$ analysis. Reactive oxygen species generation was also measured in human erythrocytes incubated in the presence of quinine with and without microsomes.

Results: The metabolites 3-hydroxyquinine, 2'-oxoquininone, and O-desmethylquinine were observed after incubation with CYPs 3A4 (3-hydroxyquinine and 2'-oxoquininone) and 2D6 (O-desmethylquinine). In addition, multiple hydroxylations were observed both on the quinoline core and the quinuclidine ring system. Of the five primary abundance CYPs tested, 3A4, 2D6, 2C9, and 2C19 all demonstrated activity toward quinine, while $1 \mathrm{~A} 2$ did not. Further, quinine produced robust dose-dependent oxidative stress in human erythrocytes in the presence of microsomes.
\end{abstract}

Conclusions: Taken in context, these data suggest a CYP-mediated link between quinine metabolism and the poorly understood haemolytic condition known as blackwater fever, often associated with quinine ingestion.

Keywords: Quinine, Blackwater fever, Metabolism, CYP450, Metabolite identification

\section{Background}

Usage of the alkaloid anti-malarial agent, quinine to treat resistant strains of Plasmodium falciparum and as an intravenous treatment for severe malaria has increased over the past few decades [1-4]. Several in vitro and in vivo studies have reported numerous metabolites of quinine, including 3-hydroxyquinine, O-desmethylquinine, and 2'-oxoquininone and speculations have been made concerning their origins. However, the CYP 450 enzymes responsible for their formation have yet to be identified [4-8]. For example, inhibition studies have indicated that

\footnotetext{
* Correspondence: brandon.s.pybus.mil@mail.mil

'Department of Drug Development, Division of Experimental Therapeutics, Walter Reed Army Institute of Research, Silver Spring, MD 20910, USA Full list of author information is available at the end of the article
}

3-hydroxyquinine production may be CYP $3 \mathrm{~A} 4$ mediated $[4,9,10]$. However, Wanwimolruk et al. have shown that, while pretreatment with the CYP 3A4 inducer, rifampin seemed to enhance quinine clearance, no appreciable changes in 3-hydroxyquinine formation were noted after such treatment $[4,8]$. In order to address some of these inconsistencies in the literature, a set of in vitro experiments were undertaken to unambiguously assign the metabolites of quinine produced by each of the five primary abundance CYPs (1A2, 3A4, 2C9, 2C19, and 2D6).

Quinine treatment is associated with several adverse events which may have a significant metabolism component. One of the least understood and most severe is blackwater fever (BWF), a haemolytic disorder characterized by massive amounts of haemoglobin in the urine.

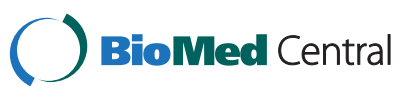


BWF has long been associated with falciparum malaria and irregular quinine ingestion [11]. Although quinine is not traditionally thought of as an oxidant drug, recent data suggest a link between quinine ingestion, glucose-6-phosphate dehydrogenase (G6PD) deficiency, malaria infection, and the occurrence of BWF [12,13]. Further, Bloom et al. reported that after incubation with microsomes and quinine, reduced glutathione (GSH) depletion was comparable to that of primaquine [14]. It is reasonable to assume that the metabolic formation of quinine, quinolones, could add low levels of oxidative stress, which combined with the background oxidative environment of a parasitized G6PD-deficient erythrocyte, may lead to the haemolytic response associated with BWF. To this end, attempts to identify novel metabolites, quinolone or otherwise, which could produce oxidative stress were also made in the present work.

\section{Methods}

\section{Chemicals used}

Chemicals used were: quinine (Sigma, St Louis, MO, USA, \# 6119-47-7), nicotinamide adenine dinucleotide phosphate, oxidized form (NADP) (Sigma, \# 077K7000), acetonitrile (Fisher Scientific, Waltham, MA, USA, \#972970), glucose-6-phosphate (G6P) (Sigma, \# 046K3779), glucose6-phosphate dehydrogenase (G6PD) (Sigma, \# 068K3795), and magnesium chloride $\left(\mathrm{MgCl}_{2}\right)$ (Sigma, \#102K0154). Mobile phases were made with HPLC grade water, acetonitrile, and formic acid. The ROS probe 2, 7-dichlorofluorescein diacetate (DCFDA) was obtained from Molecular Probes/Invitrogen, (Eugene, OR, USA).

\section{CYP incubations}

In vitro metabolism studies with isoenzymes were conducted according to the manufacturer's instructions (BD Gentest, San Jose, CA, USA). Briefly, the procedure was as follows. A $30 \mu \mathrm{l}$ aliquot of $5 \mathrm{mg} / \mathrm{ml}$ isoenzyme, either CYP 1A2, 3A4, 2C9, 2C19, or 2D6, was mixed with NADPH regeneration system $\mathrm{A}(50 \mu \mathrm{l})$ and $\mathrm{B}(10 \mu \mathrm{l})$, and

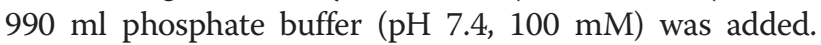
The solution was mixed gently by pipetting and incubated at $37^{\circ} \mathrm{C}$ for about $2 \mathrm{~min}$, and then the test compound, quinine (10 $\mu \mathrm{M}$ final concentration), was added. A portion of the mixture $(120 \mu \mathrm{l})$ was then collected at several time points $(0,60 \mathrm{~min})$ followed by quenching with an equal volume of acetonitrile. The samples were vortexed for $30 \mathrm{sec}$, and centrifuged at $13,600 \mathrm{~g}$ at $4^{\circ} \mathrm{C}$ for $10 \mathrm{~min}$. Supernatant was collected and loaded onto 96-well plates $(200 \mu \mathrm{l} /$ well $)$ for LC-MS analysis.

\section{Accurate mass metabolite identification}

Quinine samples were analysed using a Waters (Milford, MA, USA) Acquity UPLC system coupled to a Xevo Q-ToF mass spectrometer equipped with a standard electrospray ionization source. Chromatographic separations were achieved using a Waters Acquity BEH C18 $1.7 \mu \mathrm{m} 2.1$ $\mathrm{mm} \times 100 \mathrm{~mm}$ column with a 2 to $98 \%$ acetonitrile gradient over $6.10 \mathrm{~min}$ at a flow rate of $0.70 \mathrm{~mL} / \mathrm{min}$. Mobile phase A consisted of $10 \mathrm{mM}$ ammonium bicarbonate and mobile phase $\mathrm{B}$ consisted of acetonitrile. The gradient consisted of phase B increasing from 2 to $60 \%$ in the time period of 0 to $2.9 \mathrm{~min}$, followed by 60 to $98 \%$ from 2.9 to $4.7 \mathrm{~min}$, holding at $98 \% \mathrm{~B}$ from 4.7 to $5.2 \mathrm{~min}$, and then returning to $2 \% \mathrm{~B}$ from 5.2 to $6.1 \mathrm{~min}$. MS conditions were optimized for quinine detection in the positive electrospray mode with the corresponding instrumental parameters: capillary $3 \mathrm{kV}$, sampling cone $35 \mathrm{~V}$, extraction cone $4 \mathrm{~V}$, source temperature $120^{\circ} \mathrm{C}$, desolvation temperature $550^{\circ} \mathrm{C}$, cone gas flow $30 \mathrm{~L} / \mathrm{Hr}$, and desolvation gas flow $1,000 \mathrm{~L} / \mathrm{hr}$. Low energy MS scans were conducted using a collision energy of $6 \mathrm{~V}$. Quinine fragments were produced using the $\mathrm{MS}^{\mathrm{E}}$ mode with a collision energy ramp from 30-35 V. Quinine metabolites were indentified and analysed using Waters Metabolynx software, $\mathrm{MS}^{\mathrm{E}}$, and MS/MS analysis.

\section{Reactive oxygen species (ROS) formation kinetics assay}

The intra-erythrocytic formation of ROS was monitored in real-time with 2'7'- dichlorofluorescein diacetate (DCFDA), a fluorescent ROS probe as described earlier [15]. Human erythrocytes that had been collected in citrate phosphate anticoagulant were used. The erythrocytes were washed twice with $0.9 \%$ saline and suspended in PBSG at a haematocrit of $10 \%$. A $60 \mathrm{mM}$ stock of DCFDA was prepared in DMSO and added to the erythrocytes suspension to obtain the final concentration of $600 \mathrm{mM}$. The suspension containing $600 \mathrm{mM}$ of DCFDA was incubated at $37^{\circ} \mathrm{C}$ for $20 \mathrm{~min}$ and centrifuged at 1,000 $\mathrm{g}$ for $5 \mathrm{~min}$. The pellet of DCFDA-loaded erythrocytes was suspended in PBSG to 50\% haematocrit and used for kinetic ROS formation assay. The microsomal metabolism-linked assay was directly set up in a clear flatbottomed, 96-well microplate. The reaction mixture contained $40 \mathrm{ml}$ of DCFDA-loaded erythrocytes, $40 \mathrm{ml}$ NADPH regeneration cocktail $\left(0.8 \mathrm{mmol} \mathrm{NADP}{ }^{+}, 5 \mathrm{mmol}\right.$ G6P, $3 \mathrm{mmol} \mathrm{MgCl}_{2}$ and 0.2 units G6PD), $40 \mathrm{ml} \mathrm{KCl} \mathrm{(31}$ $\mathrm{mmol}$ ), $10 \mathrm{ml}$ of pooled human liver microsomes (25 pmol CYP content), $2 \mathrm{ml}$ of quinine (concentration as presented below) and potassium phosphate buffer $(100 \mathrm{mM}, \mathrm{pH}$ 7.4), to make up the final volume to $200 \mathrm{ml}$. The controls without drug (with and without microsomes) were also set up simultaneously. Each assay was set up at quadruplicate. The plate was immediately placed in a microplate reader programmed to kinetic measurement of fluorescence (excitation $488 \mathrm{~nm}$ and emission $535 \mathrm{~nm}$ ) for $2 \mathrm{hr}$ with 5min time intervals. The results are presented as increase in time-dependent fluorescence by quinine with and without microsomes. 


\section{Results}

In order to determine the specific metabolites likely to be formed physiologically by each of the five primary abundance CYPs (1A2, 3A4, 2C9, 2C19, and 2D6), 1-hr incubations with quinine were conducted and the subsequent metabolites formed were analysed by accurate mass UPLC-MS ${ }^{\mathrm{E}}$. Table 1 summarizes the metabolites observed with each CYP and Figures 1 and 2 show the corresponding $\mathrm{MS}^{\mathrm{E}}$ spectra for each metabolite. In general, four types of metabolites were observed; hydroxylations on the quinoline core, hydroxylations on the quinuclidine ring, demethylations, and ketones. Further, at the concentrations chosen in this study, 3A4 metabolized quinine most extensively, followed by 2C19, 2C9, and 2D6. CYP $1 \mathrm{~A} 2$ showed very little appreciable activity towards quinine, with only trace amounts of a demethylated metabolite being observed after $60 \mathrm{~min}$. Assignments of localization for each biotransformation to either the quinoline core or quinuclidine ring were made by $M S^{\mathrm{E}}$ fragmentation. Specific variants of each subtype of metabolite observed are discussed in more detail below.

\section{Modifications on the quinuclidine ring}

$\mathrm{MS}^{\mathrm{E}}$ analysis of the quinine metabolites detected revealed that two portions of quinine were modified: the quinuclidine ring and quinoline core. This section will focus on the quinuclidine ring and the results for each ring metabolite are illustrated in Figure 1. The $\mathrm{MS}^{\mathrm{E}}$ fragmentation pattern of unmodified quinine is illustrated in Figure $1 \mathrm{~A}$ and shows the different fragmentations of quinine produced upon $\mathrm{MS}^{\mathrm{E}}$ fragmentation. These fragments encompassed the entire molecule and allowed for the localization of chemical modifications to quinine.

After incubation with CYP 3A4, multiple metabolites were observed with an $m / z$ of 341.18. As represented in
Table 1, the predominant metabolite was observed at $2.85 \mathrm{~min} . \mathrm{MS}^{\mathrm{E}}$ fragmentation showed mass changes of + $16 \mathrm{Da}$ to quinuclidine ring fragments which was consistent with the addition of a hydroxyl group (Figure 1B). Given the relative intensity of this metabolite, it was tentatively assigned as 3-hydroxyquinine. This metabolite was observed to a lesser extent after incubation with CYPs 2C9, 2C19, and 2D6. Two other metabolites with the same observed $\mathrm{m} / z$ (341.18), retention times of 2.69 and $2.94 \mathrm{~min}$, and $\mathrm{MS}^{\mathrm{E}}$ fragmentation consistent with the addition of a hydroxyl group on the quinuclidine ring (as described above) were noted after incubation with all CYPs tested except 1A2 (data not shown). In addition to single hydroxylations of the quinuclidine ring, incubations with CYP 3A4 also resulted in the appearance of a metabolite with retention time of $2.41 \mathrm{~min}$ and an observed $m / z$ of 357.18 . The +32 Da shift was localized to quinuclidine ring fragments as illustrated in Figure $1 \mathrm{C}$.

In addition to mass changes consistent with hydroxylations, one metabolite appeared at $3.12 \mathrm{~min}$ with an observed $\mathrm{m} / \mathrm{z}$ of 339.17 . This metabolite appeared with CYP 3A4 and 2C19 quinine incubations. The $+14 \mathrm{Da}$ mass increase is consistent with the addition of a hydroxyl followed by desaturation to the corresponding ketone. The $\mathrm{MS}^{\mathrm{E}}$ fragmentation pattern for this metabolite is shown in Figure 1D and indicates a mass change in one quinuclidine ring fragment $(\mathrm{m} / z$ 321.16). This fragmentation pattern is consistent with a ketone-forming alpha to the nitrogen of the quinuclidine ring. Several other metabolites with an $\mathrm{m} / z$ of 339.17 were observed, however, these were not of ample intensity to make a definitive determination as to the location of these modifications. It should be noted that this ketone is unlikely to be on the quinoline core as this modification would have

Table 1 Quinine metabolites formed upon CYP 3A4, 2D6, 2C19, 2 C9 and 1 A2 incubation Shown are the metabolites observed with each corresponding CYP

\begin{tabular}{|c|c|c|c|c|c|c|c|c|c|c|c|c|}
\hline \multirow[b]{2}{*}{ Metabolite } & \multicolumn{5}{|c|}{ Metabolite observed } & \multicolumn{2}{|c|}{ MS information } & \multicolumn{5}{|c|}{ Relative $\%$ metabolite present } \\
\hline & $3 A 4$ & 2D6 & $2 C 19$ & $2 C 9$ & $1 \mathrm{A2}$ & $m / z$ & $T_{r}(\min )$ & $3 A 4$ & 2D6 & $2 \mathrm{C} 19$ & $2 C 9$ & $1 \mathrm{A2}$ \\
\hline Parent & + & + & + & + & + & 325.19 & 3.6 & 49.04 & 95.19 & 78.22 & 93.90 & 99.31 \\
\hline Ketone & + & - & + & - & - & 339.17 & 3.12 & 0.27 & 0.00 & 0.09 & 0.00 & 0.00 \\
\hline Hydroxylation & + & + & + & + & - & 341.18 & 2.69 & 0.43 & 0.66 & 1.22 & 0.06 & 0.00 \\
\hline Hydroxylation & + & + & + & + & - & 341.18 & 2.85 & 44.88 & 0.98 & 8.36 & 0.72 & 0.00 \\
\hline Hydroxylation & + & + & + & + & - & 341.18 & 2.94 & 2.22 & 1.47 & 10.89 & 5.20 & 0.00 \\
\hline Hydroxylation/Ketone tautomers & + & - & + & + & + & 341.18 & 3.14 & 0.32 & 0.00 & 0.01 & 0.01 & 0.06 \\
\hline Demethylation + hydroxylation & + & - & - & - & - & 327.18 & 2.48 & 0.16 & 0.00 & 0.00 & 0.00 & 0.00 \\
\hline Demethylation & + & + & + & + & + & 311.17 & 3.16 & 0.15 & 1.70 & 1.23 & 0.10 & 0.62 \\
\hline 2x Hydroxlyation & + & - & - & - & - & 357.18 & 2.57 & 1.18 & 0.00 & 0.00 & 0.00 & 0.00 \\
\hline 2x Hydroxlyation & + & - & - & - & - & 357.18 & 2.41 & 1.36 & 0.00 & 0.00 & 0.00 & 0.00 \\
\hline
\end{tabular}

The plus and minus signs indicate whether the listed metabolite was observed with each CYP after 60 minute incubations. The corresponding $m / z$ values observed and retention times $\left(t_{r}\right)$ for each metabolite are listed. The relative $\%$ of each metabolite formed is also listed. 


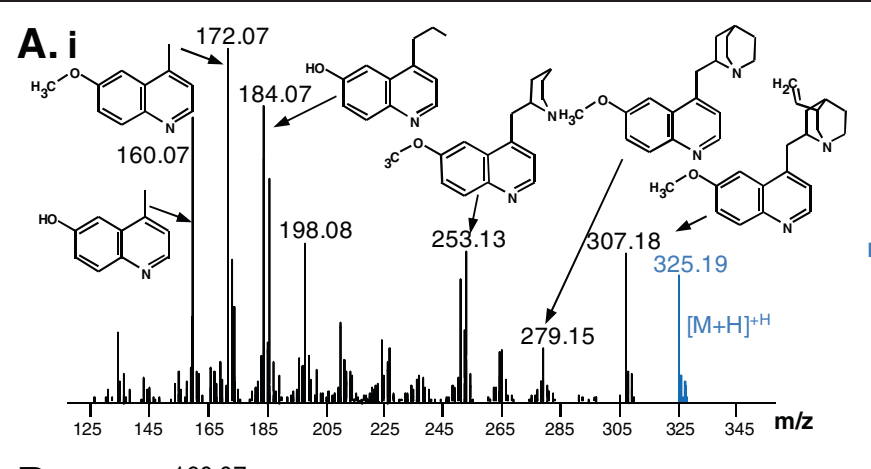<smiles>C=C1CCCN=C1C(O)c1ccnc2ccc(OC)cc12</smiles>

Observed m/z: 325.19

Mass error: $1.6 \mathrm{ppm}$

B. $\mathbf{i}$

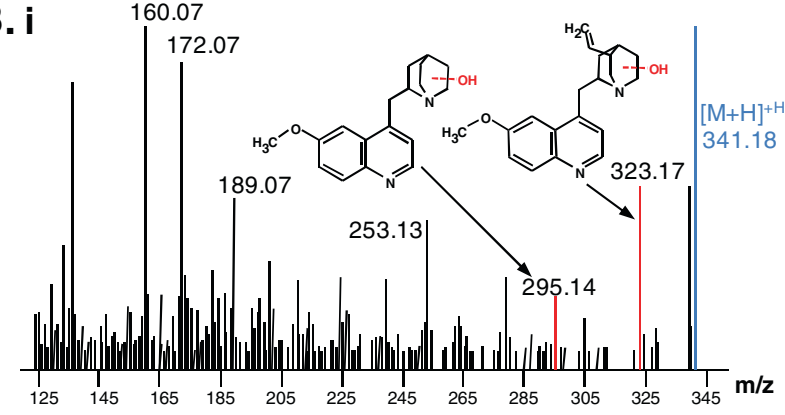

C. $\mathrm{i}$

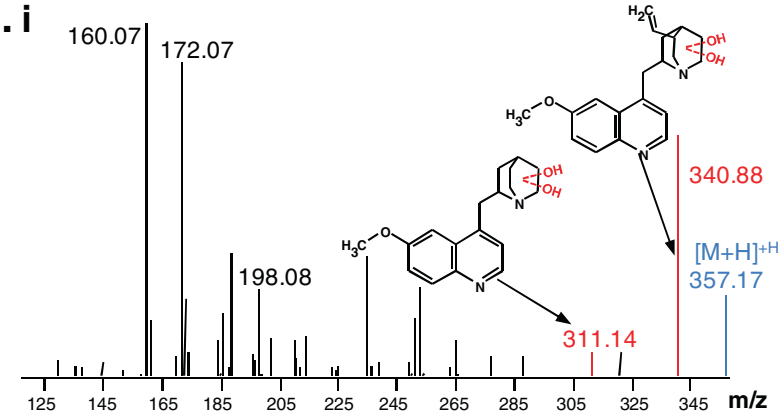

D. $\mathbf{i}$

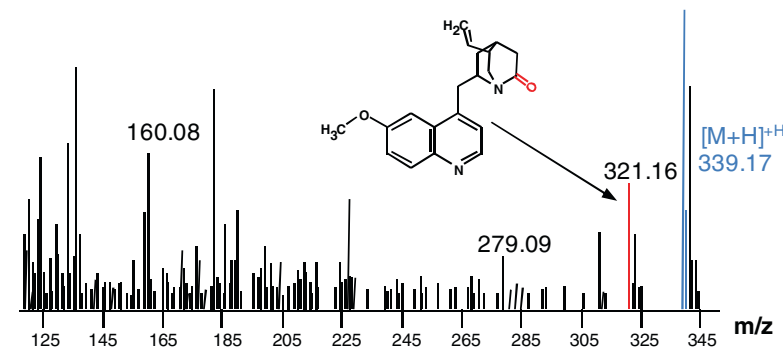

ii Tentative Metabolite<smiles></smiles>

Observed m/z: $\mathbf{3 4 1 . 1 8}$

Mass error: $0.0 \mathrm{ppm}$

CYP: 3A4, 2D6, 2C9, 2C19

ii Tentative Metabolite

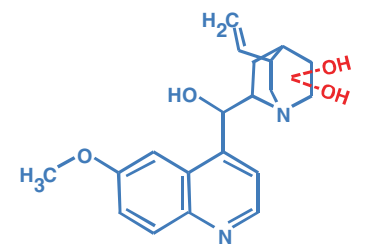

Observed m/z: 357.17

Mass error: $2.2 \mathrm{ppm}$ CYP: $3 A 4$

ii Tentative Metabolite<smiles>C=CC1CCC(=O)N1C(O)C(O)c1ccnc2ccc(OC)cc12</smiles>

Observed m/z: 339.17 Mass error: $-0.4 \mathrm{ppm}$ CYP: 3A4, 2 C19

Figure $1 \mathbf{M S}^{\mathrm{E}}$ identification of quinine quinuclidine ring metabolites. Shown in panels $\mathbf{A}-\mathbf{D}$ are the corresponding $\mathrm{MS}^{\mathrm{E}}$ spectra (i) for quinine and each quinine quinuclidine ring metabolite. The $\mathrm{m} / \mathrm{z}$ values for each ion are indicated as well as the assigned quinine fragment. The assigned metabolite structures are shown in (ii). The observed parent ion $\mathrm{m} / \mathrm{z}$, mass error, and CYPs responsible for production of each metabolite are indicated under each structure.

an expected $\mathrm{m} / \mathrm{z}$ of 341.18 . The ketone formation observed must occur somewhere on the quinuclidine ring.

\section{Modifications on the quinoline core}

The quinoline core of quinine was also modified upon incubation with the various CYPs. Incubations with either CYP 3A4, 2C19, or 2C9 resulted in the appearance of a metabolite with a retention time of $3.06 \mathrm{~min}$ and an observed $m / z$ of 341.17 . The $\mathrm{MS}^{\mathrm{E}}$ fragmentation pattern for this metabolite is shown in Figure $2 \mathrm{~A}$ and the +16 Da mass shift is consistent with the addition of a hydroxyl group to the quinoline core. This metabolite was tentatively assigned as the 2' hydroxylated form of quinine. It should be noted that by accurate mass and $M S^{\mathrm{E}}$ 


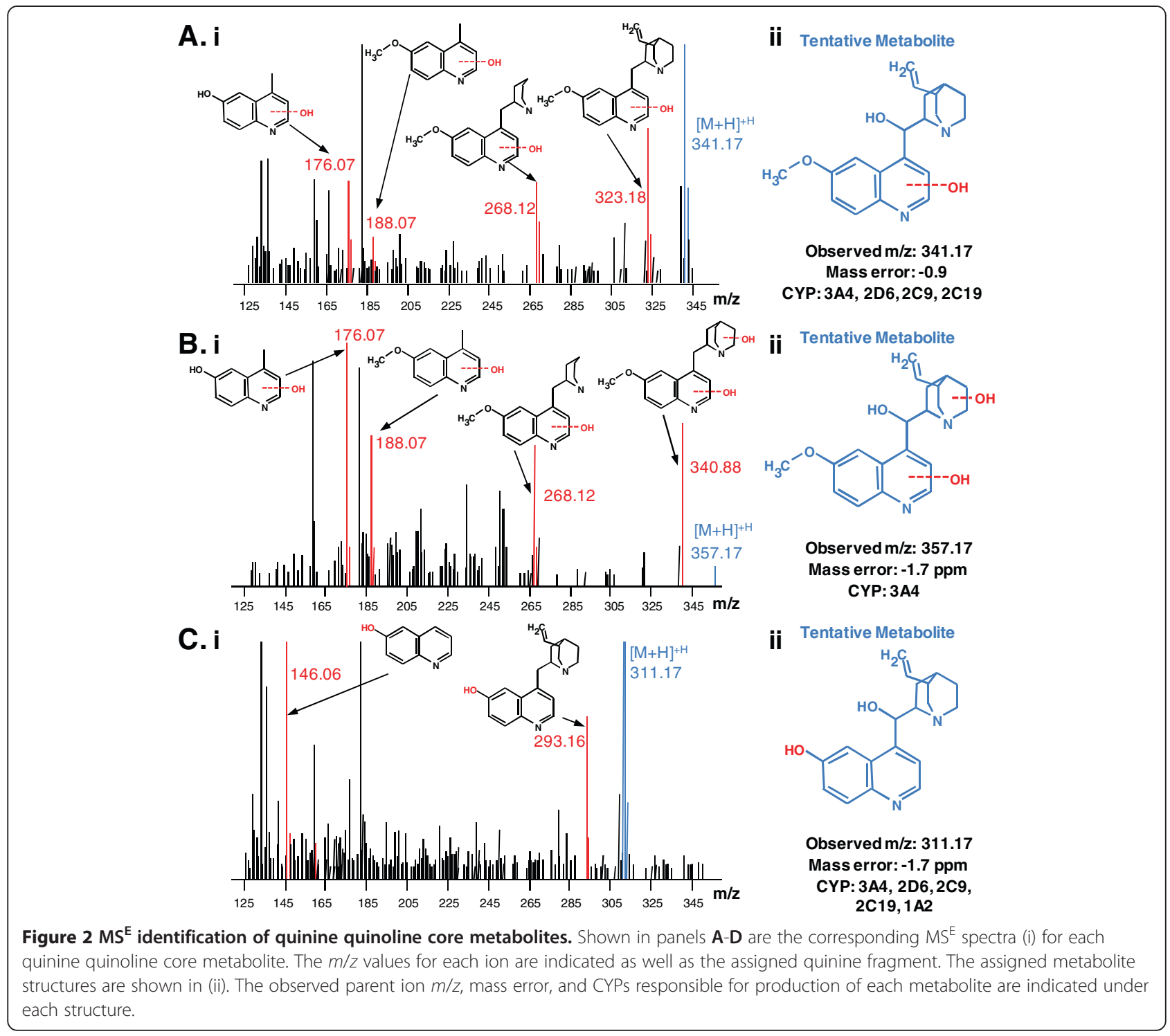

fragmentation, this metabolite is indistinguishable from its expected tautomer ( 2 '-oxoquininone). Further, a metabolite appeared after CYP 3A4 incubation at a retention time of 2.57 min with an $\mathrm{m} / \mathrm{z}$ of 357.17 consistent with the addition of two hydroxyl groups (+ $32 \mathrm{Da})$. $\mathrm{MS}^{\mathrm{E}}$ fragmentation patterns indicated that one of these hydroxylations occurs on the quinoline core, with the other occurring on the quinuclidine ring as shown in Figure 2B. In addition to the hydroxylated metabolites described above, all CYPs tested generated a metabolite with a retention time of $2.48 \mathrm{~min}$ and an observed $\mathrm{m} / \mathrm{z}$ of 311.17. This peak was assigned to the structure in which the 6' methoxy has been demethylated (-14 Da). $\mathrm{MS}^{\mathrm{E}}$ fragmentation of this metabolite is shown in Figure $2 \mathrm{C}$ and confirms the loss of a methyl group to all fragments associated with the quinoline core. Although CYP 2D6 generated the most of this metabolite under these experimental conditions, all CYPs tested generated detectable amounts of this metabolite. It should be noted that the primary purpose of the present study was to identify the number and type of CYPs involved in quinine clearance and not to attribute relative contributions from each in vivo. Further, as with 2' hydroxylation, this metabolite is indistinguishable by accurate mass or $\mathrm{MS}^{\mathrm{E}}$ fragmentation from its expected tautomer (6'-oxoquininone).

\section{ROS formation kinetics assay}

Having established from CYP incubations and MS analysis that quinine forms metabolites that have the potential to redox cycle and create oxidative stress, the ability of quinine to produce ROS was assayed and the results illustrated in Figure 3. Quinine was assayed in normal human erythrocytes in the presence and absence of 


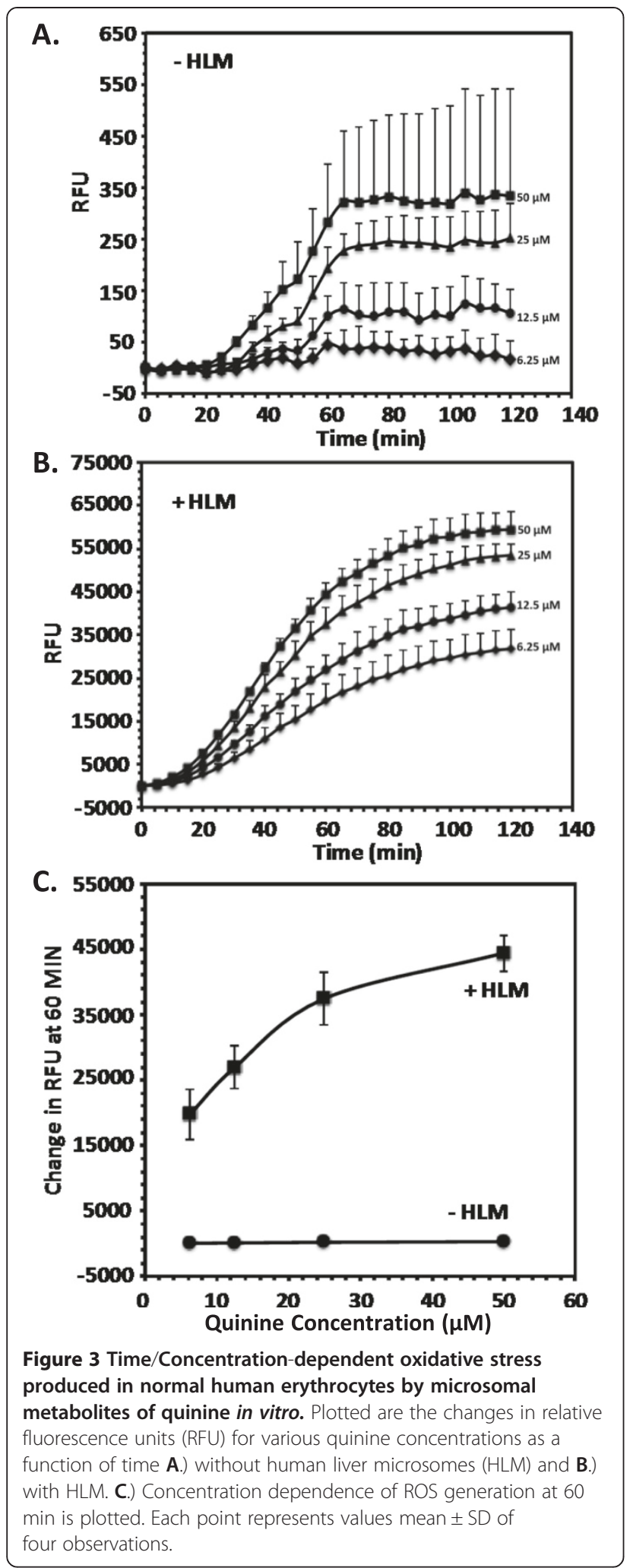

pooled human liver microsomes. Quinine did not produce noticeable oxidative stress in normal human erythrocytes without microsomes as indicated by no significant increase in DCFDA fluorescence. However, incubation of human erythrocytes with quinine in presence of human liver microsomes produced robust reactive oxidative stress as indicated by time-dependent increase in DCFDA fluorescence. These observations indicate that quinine metabolites generated through CYP-mediated pathways are responsible for producing intracellular oxidative stress in the erythrocytes. Further, the increase in ROS production by the microsomal metabolites of quinine was observed to be concentration dependent (Figure 3).

\section{Conclusions}

The cinchona alkaloid anti-malarial agent, quinine is extensively metabolized in vivo, and many of the resulting metabolites have been reported both in urine and plasma $[4,7,8,16]$. While these studies have largely used synthetic metabolites of the diasteriomer quinidine as reference standards for HPLC identification rather than direct observation via accurate mass MS, the consensus assignments are not in question. However, ambiguity remains concerning the specific CYP pathways responsible for the production of the major metabolites, and therefore the primary clearance routes of this drug. For example, Wanwimolruk et al. observed that both cigarette smoking and rifampin pretreatment enhance the clearance of quinine, suggestive of important roles for both CYP3A and 1A families in quinine clearance. They further noted that neither observation correlated with a significant increase in the ratio of 3-hydroxyquinine, a major metabolite, in the urine $[4,8]$. Other metabolites have been observed in urine such as 2'-oxoquininone and $\mathrm{O}$-desmethylquinine which have not been assigned to specific CYP pathways $[6,7,16]$.

In the present study, CYP 3A4 was identified as predominantly responsible for the production of 3hydroxyquinine, and a second hydroxylation localized to the quinoline core. To date, the only hydroxylation reported on the quinoline core occurred at the 2' position, therefore this metabolite most likely corresponds to 2'hydroxyquinine. It is important to note that the tautomeric quinolone form of this metabolite is isobaric, but is assumed to exist for this metabolite and all others in which hydroxylation occurs on the quinoline core. Three other keto metabolites were observed after incubation with CYP 3A4, one of which localized to the quinuclidine ring, as determined by $\mathrm{MS}^{\mathrm{E}}$, and the others which had fragmentation patterns with insufficient signal intensity for accurate localization. However, the $m / z$ associated with each of these ketone metabolites was only consistent with addition to the quinuclidine ring. CYP3A4 was also found to mediate the production of two doubly hydroxylated metabolites, one in which hydroxylation occurred both on the quinoline core and the quinuclidine ring (2', 3 -dihydroxyquinine is the most likely possibility for 


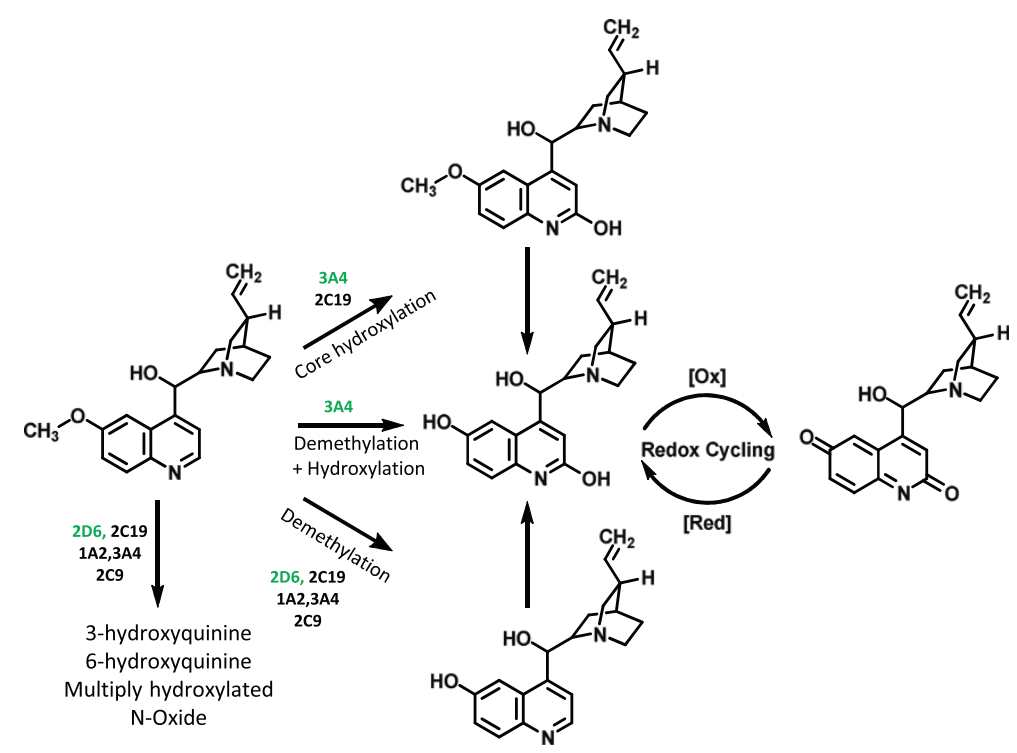

Figure 4 Summary of quinine metabolism. Shown are the identified metabolites of quinine and their predicted redox active forms. The CYPs responsible for each transformation are indicated for each pathway and the CYPs primarily responsible for each transformation are indicated in green. Several other metabolites are listed for which the structures are not shown (3-hydroxyquinine, 6-hydroxyquinine, N-oxide, and multiply hydroxylated).

assignment) and a second with two hydroxylations occurring on the quinuclidine ring. Given the abundance in plasma of 3-hydroxyquinine, it is reasonable to assume that hydroxylation at the 3 position accounts for one of these, while the other hydroxylation may occur alpha to the nitrogen at the 6 position on the quinuclidine ring. This would correspond to the reported dihydroxy nonphenolic metabolite reported by Brodie et al. [16]. While O-demethylation was observed after incubation with all five CYPs tested, under these experimental conditions CYP 2D6 produced the most of this metabolite followed by $2 \mathrm{C} 19,3 \mathrm{~A} 4,1 \mathrm{~A} 2$, and $2 \mathrm{C} 9$ respectively. Further, CYP 3A4 was found to catalyze hydroxylation of this metabolite, presumably at the 2' position for the reasons discussed above. Lastly, two other hydroxylated species were observed, which were generated at various levels with CYPs $3 \mathrm{~A} 4,2 \mathrm{D} 6,2 \mathrm{C} 9$, and 2C19. As stated above, one of these would presumably be the substitution alpha to the quinuclidine nitrogen atom reported by Brodie et al., while the other would seem to be an as yet unidentified metabolite [16]. A schematic representation of the major phase I metabolic pathways elucidated from this work is provided (Figure 4).

Bloom et al. reported GSH depletion after incubation with microsomes and quinine, comparable to levels of known oxidant drugs [14]. Interestingly, recent data suggest quinolone drugs have the capacity to generate reactive oxygen species in the presence of human liver microsomes, and in fact have been shown to reduce glutathione levels in rat liver $[17,18]$. Fluoroquinolone drugs are also often associated with a haemolytic anaemia which may in some ways be similar to the haemolysis seen with blackwater fever [19-23]. Further, a strong correlation between BWF and G6PD deficiency has been established $[12,13]$. Oxidant molecules cause accelerated generation and accumulation of reactive oxygen intermediates (superoxide radical, hydroxyl radical and hydrogen peroxide) in the erythrocytes [24]. In the present study, it was clearly shown that products of CYP-mediated quinine metabolism generate robust, dose-dependent oxidative stress in human erythrocytes (Figure 3). This may in part be due to redox cycling of the quinone species observed after incubation with CYP 3A4 (Figure 4). This would become increasingly important in individuals with already high backgrounds of oxidative stress, such as those infected with malaria or with a genetic deficiency such as G6PD deficiency. It is reasonable to speculate that quinine metabolites rather than quinine itself are at least partially responsible for the haemolytic oxidative stress associated with BWF. While the pathogenesis of BWF is incompletely understood, the metabolite identification outlined here identifies a class of molecules that warrant further investigation, specifically regarding the ability of these species to generate oxidative stress in the erythrocyte.

\section{Abbreviations}

BWF: Blackwater fever; CYP: Cytochrome P450; DCFDA: 2,7-Dichlorofluorescein diacetate; HLM: Pooled human liver microsomes; MS: Mass spectrometry; PBSG: Phosphate buffered saline with glucose; RFU: Relative fluorescence units; ROS: Reactive oxygen species; UPLC: Ultra performance liquid chromatography.

\section{Competing interests}

The authors have declared that they have no competing interests. 


\section{Authors' contributions}

SRM, XJ, GDS, JCS, VM and BSP participated in research design; SRM, XJ, TB, NM, BLT and RS conducted experiments; VM contributed new reagents or analytic tools; SRM, XJ and BSP performed data analysis; SRM, XJ, GDS, JCS, $B L T, R S, G A R, R J S$ and BSP wrote or contributed to the writing of the manuscript. All authors read and approved the final manuscript.

\section{Acknowledgement}

Disclaimer

The views, opinions and/or findings contained in this presentation are those of the authors and do not necessarily reflect the views of the US Department of Defense or the Australian Defence Force and should not be construed as an official DoD/Army/Australian Defence Force position, policy or decision unless so designated by other documentation. No official endorsement should be made.

\section{Author details}

'Department of Drug Development, Division of Experimental Therapeutics, Walter Reed Army Institute of Research, Silver Spring, MD 20910, USA.

${ }^{2}$ Australian Army Malaria Institute, Gallipoli BarracksEnoggera, 4051QLD, Australia. ${ }^{3}$ National Center for Natural Products Research, School of Pharmacy, University of Mississippi, Oxford, MS 38677, USA.

Received: 19 April 2013 Accepted: 11 June 2013

Published: 21 June 2013

\section{References}

1. Mathew JL: Artemisinin derivatives versus quinine for severe malaria in children: a systematic review and meta-analysis. Indian Pediatr 2010, 47:423-428.

2. Praygod G, de Frey A, Eisenhut M: Artemisinin derivatives versus quinine in treating severe malaria in children: a systematic review. Malar J 2008 7:210.

3. Sinclair D, Donegan $S$, Isba R, Lalloo DG: Artesunate versus quinine for treating severe malaria. Cochrane Database Syst Rev 2012, 6, CD005967.

4. Wanwimolruk S, Wong SM, Zhang H, Coville PF, Walker RJ: Metabolism of quinine in man: identification of a major metabolite, and effects of smoking and rifampicin pretreatment. J Pharm Pharmacol 1995, 47:957-963.

5. Bannon P, Yu P, Cook JM, Roy L, Villeneuve JP: Identification of quinine metabolites in urine after oral dosing in humans. J Chromatogr B: Biomed Sci App/ 1998, 715:387-393.

6. Bolaji $\mathrm{OO}$, Babalola CP, Dixon PA: Characterization of the principal metabolite of quinine in human urine by $1 \mathrm{H}-$ n.m.r. spectroscopy. Xenobiotica 1991, 21:447-450

7. Liddle C, Graham GG, Christopher RK, Bhuwapathanapun S, Duffield AM: Identification of new urinary metabolites in man of quinine using methane chemical ionization gas chromatography-mass spectrometry. Xenobiotica 1981, 11:81-87.

8. Wanwimolruk S, Kang W, Coville PF, Viriyayudhakorn S, Thitiarchakul S: Marked enhancement by rifampicin and lack of effect of isoniazid on the elimination of quinine in man. Br J Clin Pharmacol 1995, 40:87-91.

9. Zhao XJ, Ishizaki T: The In vitro hepatic metabolism of quinine in mice, rats and dogs: comparison with human liver microsomes. J Pharmacol Exp Ther 1997, 283:1168-1176.

10. Zhao XJ, Yokoyama H, Chiba K, Wanwimolruk S, Ishizaki T: Identification of human cytochrome P450 isoforms involved in the 3-hydroxylation of quinine by human live microsomes and nine recombinant human cytochromes P450. J Pharmacol Exp Ther 1996, 279:1327-1334.

11. George CR: Blackwater fever: the rise and fall of an exotic disease. J Nephrol 2009, 22(Suppl 14):120-128.

12. Tran $T H$, Day NP, Ly VC, Nguyen TH, Pham PL, Nguyen HP, Bethell DB, Dihn XS, Tran $\mathrm{TH}$, White NJ: Blackwater fever in southern Vietnam: a prospective descriptive study of 50 cases. Clin Infect Dis 1996, 23:1274-1281.

13. Hue NT, Charlieu JP, Chau TT, Day N, Farrar JJ, Hien TT, Dunstan SJ: Glucose-6-phosphate dehydrogenase (G6PD) mutations and haemoglobinuria syndrome in the Vietnamese population. Malar J 2009, 8:152.

14. Bloom KE, Brewer GJ, Magon AM, Wetterstroem N: Microsomal incubation test of potentially hemolytic drugs for glucose-6-phosphate dehydrogenase deficiency. Clin Pharmacol Ther 1983, 33:403-409.
15. Ganesan S, Chaurasiya ND, Sahu R, Walker LA, Tekwani BL: Understanding the mechanisms for metabolism-linked hemolytic toxicity of primaquine against glucose 6-phosphate dehydrogenase deficient human erythrocytes: evaluation of eryptotic pathway. Toxicology 2012, 294:54-60.

16. Brodie BB, Baer JE, Craig LC: Metabolic products of the cinchona alkaloids in human urine. J Biol Chem 1951, 188:567-581.

17. Gurbay A, Gonthier B, Daveloose D, Favier A, Hincal F: Microsomal metabolism of ciprofloxacin generates free radicals. Free Radic Biol Med 2001, 30:1118-1121.

18. Gurbay A, Hincal F: Ciprofloxacin-induced glutathione redox status alterations in rat tissues. Drug Chem Toxicol 2004, 27:233-242.

19. Blum MD, Graham DJ, McCloskey CA: Temafloxacin syndrome: review of 95 cases. Clin Infect Dis 1994, 18:946-950.

20. Lim S, Alam MG: Ciprofloxacin-induced acute interstitial nephritis and autoimmune hemolytic anemia. Ren Fail 2003, 25:647-651.

21. Maguire RB, Stroncek DF, Gale E, Yearlsey M: Hemolytic anemia and acute renal failure associated with temafloxacin-dependent antibodies. Am J Hematol 1994, 46:363-366

22. Oh YR, Carr-Lopez SM, Probasco JM, Crawley PG: Levofloxacin-induced autoimmune hemolytic anemia. Ann Pharmacother 2003, 37:1010-1013.

23. Rubinstein E: History of quinolones and their side effects. Chemotherapy 2001, 47(Suppl 3):3-8. discussion 44-48.

24. Sivilotti ML: Oxidant stress and haemolysis of the human erythrocyte. Toxicol Rev 2004, 23:169-188.

doi:10.1186/1475-2875-12-214

Cite this article as: Marcsisin et al:: CYP450 phenotyping and metabolite identification of quinine by accurate mass

UPLC-MS analysis: a possible metabolic link to blackwater fever. Malaria Journal 2013 12:214.

\section{Submit your next manuscript to BioMed Central and take full advantage of:}

- Convenient online submission

- Thorough peer review

- No space constraints or color figure charges

- Immediate publication on acceptance

- Inclusion in PubMed, CAS, Scopus and Google Scholar

- Research which is freely available for redistribution 